\title{
Novel Low Reflective Index Fluoropolymers based Top Anti-reflective Coatings (TARC) for 193-nm Lithography
}

\author{
Tsuneo Yamashita, Takashi Hayami, Takuji Ishikawa, \\ Takashi Kanemura, Hirokazu Aoyama
}

\author{
Fundamental Research Dept. Chemical Div. Daikin Industries, Ltd., I-1 Nishi-Hitotsuya, Settsu, \\ Osaka, 566-8585 Japan
}

\begin{abstract}
Implant lithography, which has up to now utilized 365-nm (i-line) and 248-nm ( $\mathrm{KrF}$ ) light sources, must now turn to 193-nm (ArF) sources. In implant lithography, an anti-reflective material is often used to coat the resist-film. The top anti-reflective coating (abbreviated to TARC) is most often used to reduce CD swing. TARC materials must have low refractive index and water solubility. The TARC materials for used 193-nm use must have very low reflective index and alternatives to perfluorooctylsulfonic acid (PFOS) and perfluorooctanoic acid (PFOA) must be found. We synthesized some novel fluorinated amorphous polymers as 193-nm TARC candidates. Their fundamental properties were characterized, such as transparency and reflective index at 193-nm (wavelength) along with their solubility in water and a standard alkaline developer. High transparency, i.e., $\mathrm{k}$ value less than 0.01 , and very low reflective index, i.e., lower than $\mathrm{n}=1.4$ at $193-\mathrm{nm}$ wavelength are confirmed. Their dissolution behaviors are studied using the Quartz Crystal Microbalance (QCM) method. In surprise finding, we find that several of the polymers examined, those that have high fluorine content, dissolved in water. Test results show that the proposed polymers can be applied as top anti reflective coatings .

Keywords: 193-nm lithography, implant lithography, resist, fluoropolymers, Quartz Crystal Microbalance (QCM), TARC (Top Anti- $\underline{\text { Reflective }}$ Coating), low reflective index, perfluorooctylsulfonic acid (PFOS), amorphous polymers

perfluorooctylcalboxylic acid (PFOA) .
\end{abstract}

\section{Introduction}

Fluoropolymers shows high transparency from a visible light domain to the domain of vacuum ultraviolet. This is because the absorption based on electronic changes of $\mathrm{C}-\mathrm{F}$ bonds lies in the domain of vacuum ultraviolet, unlike $\mathrm{C}-\mathrm{H}$ bonds. Fluoropolymers have long been thought of as one of the best approaches to achieving high transparency at shorter wavelengths, and particular attention has paid to fluoropolymers for $157-\mathrm{nm}$ lithography. Many fluoropolymers for use as $157-\mathrm{nm}$ resist base resins have been intensively studied over the past six years [1-8].
Our previous study on the dissolution behavior of fluoropolymers $[9-12,15,16]$ showed their excellent characteristics. We have synthesized main-chain and side-chain fluorinated polymers using hexafluoropropanol (HFA) containing monomers and characterize their dissolution behavior. $193-\mathrm{nm}$ resists mainly make use of carboxylic acid as the polymer's acid group, which result in swelling during aqueous development. The HFA group has acidity similar to that of the phenol group. Consequently, HFA containing polymer had small swelling and moderate dissolution speed. 
Furthermore, a low refractive index appears to be characteristic of fluoropolymers. By the Lorentz-Lorenz formula (1), if the sum of atomic refraction is small and polymer density is low, the refractive index of polymer will be low.

$$
n_{D}=(1+2[R] / V) /(1-[R] / V)^{1 / 2}
$$

$n_{D}$ : Refractive index of polymer, $[R]$ : The sum of atomic refraction, $V$ : molecular volume $(=\mathrm{M} /$ $\rho \mathbf{M}$ : monomer molecular weight and $\rho$ :polymer density)

Unlike hydrogen or carbon, fluoride has a low atomic reflective index. By increasing the number of $\mathrm{C}-\mathrm{F}$ bonds, while retraining the same main chain structure and polymer density, we can create a resin that has a low refractive index. The optical absorption bands of a fluoropolymers lie in the short wavelength side of the vacuum ultraviolet domain; their low refractive index is relative constant against wavelength from visible light up to the vacuum ultraviolet domain. We started development of new materials for TARC use based on these excellent characteristics. Conventionally, TARC material was the mixture of PFOS or PFOA and a water-soluble polymer. . This approach, unfortunately, yields an inadequate refractive index and raises the problem of environmental damage. The need for a satisfactory alternative is urgent.

This paper introduces novel low reflective index fluoroporimers that are suitable as TARC materials. We synthesized novel fluorinated amorphous polymers, called trifluoroallyloxyether polymers, and characterize their fundamental properties, such as transparency and reflective index at 193-nm (wavelength), along with their dissolution behavior in water and $0.262 \mathrm{~N}$ TMAH.

\section{Experimental}

\subsection{Materials}

All materials were obtained from commercial suppliers and used without further purification, unless otherwise noted.

\subsubsection{Synthesis of monomer}

Trifuruoroallyloxyether monomers were prepared by the oligomerization of 2,2,3,3-tetrafluorooxetane and hexafluoropropyleneoxide as described in the literature [13] (Scheme 1). These monomers have a unique; a combination of a functional group and a perfluoro ether moiety. A functional group is

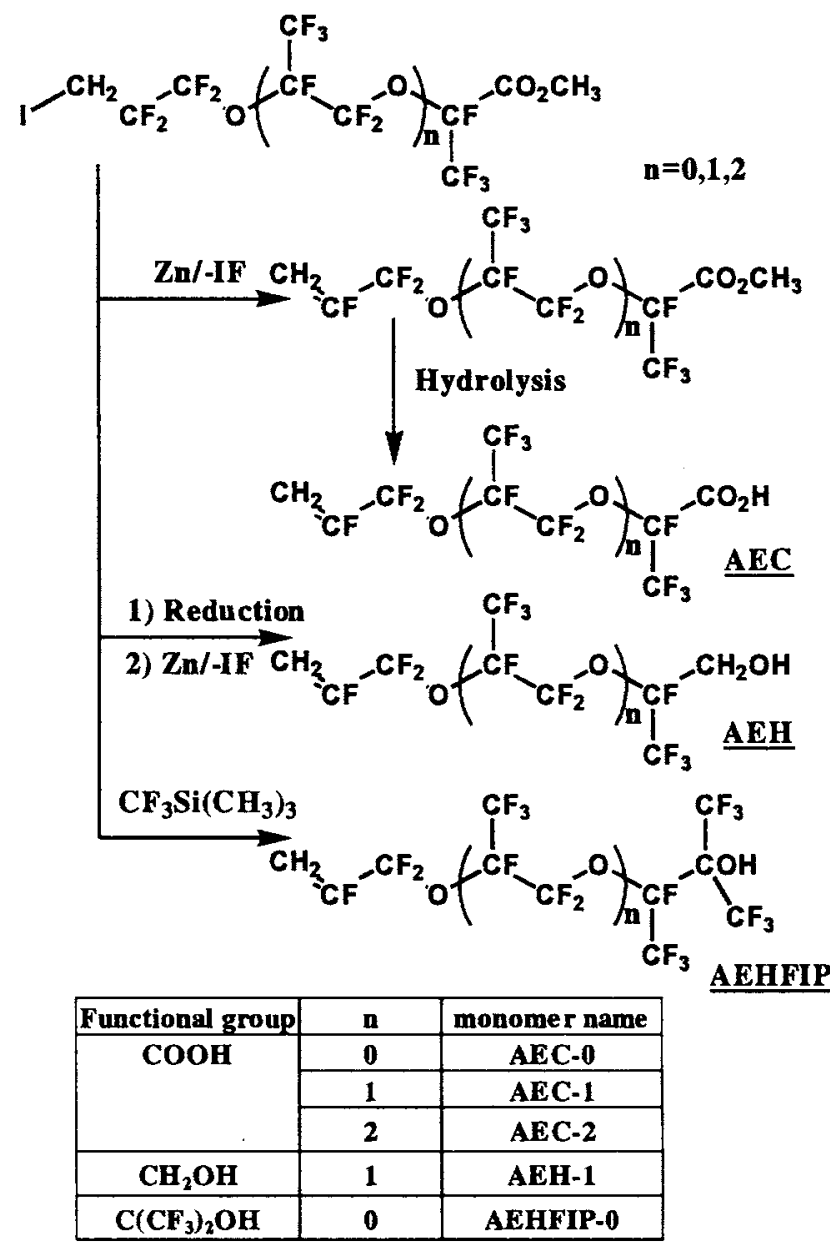

Scheme 1. Synthetic method of new trifluoroaryloxyether monomer.

convertible for calboxylic acid, alcohol, ester, etc. Moreover, fluoride content can be optimized by setting alkylchaine length. In this paper, we chose three functional groups, calboxylic acid, alcohol, and hexafluoroalcohol (HFA). The calboxylic acid (called AEC) was easily prepared by hydrolized of corresponding ester. The alcohol (AEH) was prepared from the reduction and elimination fluoride and Iodine of 1. The HFA (AEHFIP) was directly obtained by the reaction of 1 with 2 equivalents of trifluoromethyltrimethylsilane $\mathrm{CF}_{3} \mathrm{Si}\left(\mathrm{CH}_{3}\right)_{3}$ in THF at room temperature.

[2,3,3,3-tetrafluoro-2-(1,1,2,3,3,3-hex afluoro-2 -(1,1,2,3,3,3-hexafluoro-2-(1,1,2-trifluoroallyloxy) propoxy)propoxy)propanoic acid (AEC-2) ] 
Methyl

2,3,3,3-tetrafluoro-2-(1,1,2,3,3,3-hexafluoro-2-(1,1 ,2,3,3,3-hexafluoro-2-(1,1,2-trifluoroallyloxy)prop oxy)pr opoxy)propanoate was slowly dropped into $12 \mathrm{~N}-\mathrm{NaOH}$ aq at $0^{\circ} \mathrm{C}$ and stirred at room temperature for overnight. $50 \% \mathrm{H}_{2} \mathrm{SO}_{4} \mathrm{H}$ aq was slowly dropped into the reaction mixture which was kept below $20^{\circ} \mathrm{C}$. The reaction mixture was separated and collected under layer. The under layer was washed with water three times and n-hexane twice, evaporated to yield $(92 \%)$ of AEC-2 as a colorless oil.

[1,1,1,3,4,4,4-heptafluoro-3-(1,1,2-trifluoroallylox y)-2-(trifluoromethyl)butan-2-ol (AEHFIP-0)] Methyl

2,3,3,3-tetrafluoro-2-(1,1,2,2-tetrafluoro-3-iodopro poxy)propanoate was added to a suspension of dry $\mathrm{KF}$ and dry CsF in anhydrous THF under nitrogen. Trifluoromethyltrimethylsilane was slowly dropped into the mixture under nitrogen at $10^{\circ} \mathrm{C}$. The reaction mixture was stirred at $50^{\circ} \mathrm{C}$ for 3 hours. Next, water and t-butylammoniumfluoride were added to the mixture and stirred at $50^{\circ} \mathrm{C}$ for 3 hours. After reaction, $1.0 \mathrm{~N} \mathrm{HCl}$ aq was added to the mixture and extracted with diethyl ether. The combined ether extracts were washed with $1.0 \mathrm{~N}$ $\mathrm{HCl}$ aq, water, and then brine. The ether solution was dried over $\mathrm{MgSO}_{4}$, evaporated and distilled at $50^{\circ} \mathrm{C}$ at $5 \mathrm{mmHg}$ to yield (50\%) of AEHFIP-0 as a colorless oil.

\subsubsection{Polymerization}

The radical polymerization procedure was carried out at $10-30^{\circ} \mathrm{C}$ in $\mathrm{N}_{2}$ with

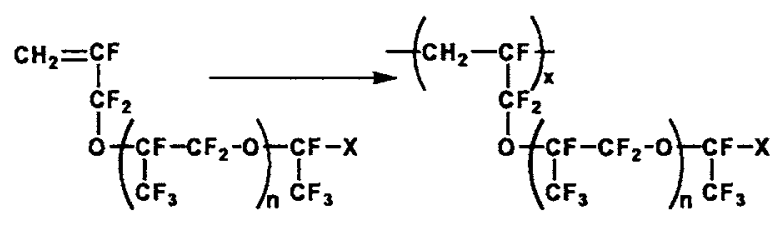

[Polymerization conditions]

In bulk under nitrogen, Room temperature for 24

$\mathrm{hr}$, Initiator; $1 \mathrm{~mol} \%$ of $(\mathrm{RfCOO})_{2}$

Scheme 2. Polymerization of trifluoroaryloxyether perfluoroalkylperoxide as the initiator in R-141b or in bulk [13]. The polymers were purified by precipitation in hexane and dried in a vacuum. The copolymers, i.e. poly (AEC/AEH), were synthesized using the same procedure. Standard copolymerization conditions are shown in (Scheme 2 ). The resulting copolymer was shown to exhibit alternate copolymerization based on $\mathrm{F}^{19}$-NMR.

\subsection{Characterization}

The polymer structures and compositions were determined from the NMR spectra. Namely, ${ }^{1} \mathrm{H}$ and ${ }^{19} \mathrm{~F}$ NMR spectra were obtained at room temperature in acetone- $\mathrm{d}_{6}$ or $\mathrm{CDCl}_{3}$ on a Brucker AC-300P FT-NMR spectrometer. The polymer structures were also confirmed by FT-IR spectra, which were captured by a PERKIN ELEMER 1760X FT-IR spectrometer.

The weight-average molecular weight $\left(M_{\mathrm{W}}\right)$ and number-average molecular weight $(M \mathrm{n})$ were determined by gel permeation chromatography (GPC, GPC-104 SHOWA DENKO K. K.) using refractive-index $(\mathrm{RI})$ data.

UV measurements at 193-nm wavelength were performed using a VUV-VASE spectroscopic ellipsometer (M-2000D, J. A. Woollam Co., Inc.). Sample films were cast on a $\mathrm{CaF}_{2}$ plate from solutions and baked at $110^{\circ} \mathrm{C}$ for 90 seconds prior to measurements. Film thickness was measured by using an interferometer. All absorbance data reported are in base 10 .

The dissolution behavior (i.e. the dissolution kinetics of the polymer film) during the development of the film was studied using a quartz crystal microbalance (QCM, Maxtek Model TM-400) in a fashion similar to a reported procedure [14]. The QCM instrumentation can, in combination with a compensated phase-locked oscillator circuit, measure the changes in film mass and motional resistance simultaneously during development. The developer used was a $0.262 \mathrm{~N}$ tetramethylammonium hydroxide (TMAH) aqueous solution (PD-523) supplied by Japan Synthetic Rubber Co., Ltd. 


\section{Results and Discussion}

3.1. Synthesis of monomer and polymer, and polymer characterization

Our approach to designing novel fluorinated amorphous polymers is to employ trifluoroallyloxyether monomer. These polymers are generally transparent, solvent-soluble, and have high alkaline-solubility. Their alkaline-solubility depends on the acidities set by the fluoroalchol and calboxylic acid group. They demonstrate improve adhesion to the substrate during the spin-coating process and wettability and solubility during the development process. Their fluorine atom content, higher than that of side-chain fluorinated polymers, avoids intermixing with the resist film and yields the low reflective index. We prepared several homo- and co-polymers from the monomer with three kinds of functional groups. The data of the prepared polymers were shown in Table 1. All polymers were prepared with high yield (over $80 \%$ ), and purification is simple to achieve by re-precipitation. Since all polymers have high enough molecular weights, they are well suited to forming thin films.

\begin{tabular}{|c|c|c|c|c|c|c|c|c|c|}
\hline \multirow{2}{*}{$\begin{array}{c}\text { Polymer } \\
\text { No. }\end{array}$} & \multicolumn{2}{|c|}{ masonetr } & \multicolumn{2}{|c|}{ conmousmet } & \multicolumn{2}{|c|}{ Ratio of molecencer } & \multirow{2}{*}{ 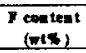 } & \multirow[t]{2}{*}{ Mn } & \multirow{2}{*}{$M w$} \\
\hline & $\bar{x}$ & 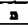 & $x$ & 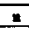 & $\mathrm{I}$ & 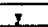 & & & \\
\hline 1 & COOH & 0 & & & 100 & & & 57k & I19t \\
\hline 2 & & & $\mathrm{CH}, \mathrm{OH}$ & 1 & 39 & 70 & 58.9 & $17 \mathrm{~K}$ & 20 \\
\hline 3 & & & & & so & so & 56.3 & $17 \mathrm{~K}$ & $20 *$ \\
\hline 4 & & & & & 35 & 45 & 56.8 & $17 \mathrm{k}$ & $2 \mathrm{~m}$ \\
\hline 5 & & & & & so & 40 & 55.4 & 271 & $40 \%$ \\
\hline 6 & & & COOH & 2 & 30 & 50 & 36.7 & 321 & $9 n$ \\
\hline 8 & & & & & 60 & 40 & 35.8 & ND & ND \\
\hline 8 & & & & & 70 & 30 & 34.8 & ND & MD \\
\hline , & & & $\mathrm{C}(\mathrm{CP})_{3)} \mathrm{OH}$ & 0 & 50 & 50 & 53.7 & $12 \mathrm{~K}$ & $\ln$ \\
\hline 10 & & & & & 40 & 60 & 60.0 & 121 & $1 \pi$ \\
\hline 11 & $\mathrm{COOH}$ & 1 & & & 100 & & 38.5 & $29 x$ & $12 \mathrm{t}$ \\
\hline 12 & $\mathrm{C}\left(\mathrm{CP}_{\mathrm{H}_{2} \mathrm{O}} \mathrm{OH}\right.$ & - & & & 100 & & 65.3 & 631: & 70 \\
\hline
\end{tabular}

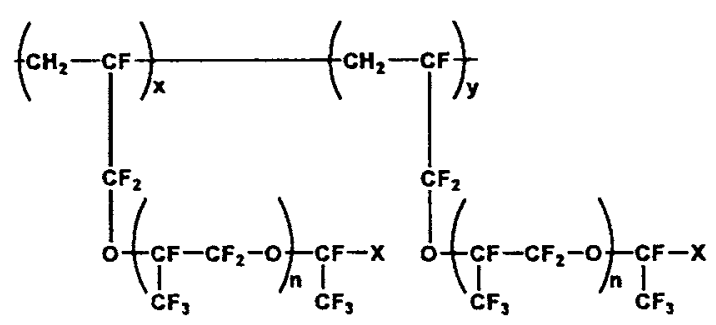

Table 1. Composition of prepared polymers

3.2. Dissolution behavior
The dissolution rates of the polymers in water and $0.262 \mathrm{~N}$ TMAH were investigated using the QCM method as shown in Table 2.

\begin{tabular}{|c|c|c|c|}
\hline \multirow{2}{*}{$\begin{array}{c}\text { Polyme r } \\
\text { No. }\end{array}$} & \multicolumn{2}{|c|}{ Diss olution rates (nm/s) } & \multirow{2}{*}{$\mathrm{pH}$} \\
\cline { 2 - 3 } & wate & 0.264 N TMAH & \\
\hline 1 & $>1000$ & $>1000$ & 1.4 \\
\cline { 2 - 4 } 3 & insoluble & 70 & \\
4 & s we lling & 79 & \\
5 & slitry soluble & $>1000$ & \\
6 & 56 & $>1000$ & 2.0 \\
7 & insoluble & & \\
8 & s we lling & 140 & \\
9 & 320 & $>1000$ & 1.7 \\
\cline { 2 - 4 } 10 & insoluble & $>1000$ & \\
11 & 120 & $>1000$ & \\
12 & soluble & & \\
\cline { 2 - 4 } 12 & insoluble & 360 & \\
\hline
\end{tabular}

Table 2. Dissolution behaviors of fluoropolymers

All polymers very rapidly dissolved in standard developer. In a surprise finding, we found that several polymers that had high fluorine content dissolved in water. The polymer containing AEC showed a low $\mathrm{pH}$ value when in aqueous solution. It is known that the $\mathrm{pH}$ of a TARC material influence the performance of the resist form, and low $\mathrm{pH}$ values are better. These excellent dissolution characteristics suggest that fluoropolymers are usable as TARC materials. Furthermore, these polymers also dissolved very well in alcohols, esters, ketones, and ethers. The vinylidene structure of the main chain and the perfluoropolyether structure of a side chain contribute to this characteristic [13]. We found that our prepared polymers could be dissolved in a solvent that does not affect the resist, coating on the resist, and then removed by the water rinse or the standard developing solution.

\subsection{Optical propaties}

TARC materials must have low refractive indices. All trifluoroaryloxyether polymers showed low reflective index and absorbance at $248-\mathrm{nm}$ and 193-nm wavelength (Table 3). The homopolymer of AEC- 0 and some copolymers of AEC-0 and AEC-2, AEH-1 or AEHFIP-0 were showed less than $n=1.5$ at $193-\mathrm{nm}$ even though they were water soluble. A key finding is that the homopolymer of AEHFIP-0 demonstrated a very low reflective 
index, $\mathrm{n}=1.39$, at $193-\mathrm{nm}$. We are now pushing the development of these polymers as TARC materials.

\begin{tabular}{c|c|c|c|c|}
\multirow{2}{*}{$\begin{array}{c}\text { Polymer } \\
\text { No. }\end{array}$} & \multicolumn{2}{|c|}{ refiactive index } & \multicolumn{2}{c|}{$k$} \\
\cline { 2 - 5 } & $0248 \mathrm{~nm}$ & $0193 \mathrm{~nm}$ & $0248 \mathrm{~nm}$ & $0103 \mathrm{~nm}$ \\
\hline 1 & 1.43 & 1.46 & $>0.001$ & $>0.001$ \\
\hline 3 & 1.41 & 1.44 & $>0.001$ & $>0.001$ \\
\hline 5 & 1.41 & 1.44 & $>0.001$ & $>0.001$ \\
\hline 8 & 1.40 & 1.44 & $>0.001$ & 0.003 \\
\hline 9 & 1.41 & 1.44 & $>0.001$ & $>0.001$ \\
\hline 10 & 1.41 & 1.44 & $>0.001$ & $>0.001$ \\
\hline 11 & & 1.42 & & $>0.001$ \\
\hline 12 & 1.37 & 1.39 & $>0.001$ & $>0.001$ \\
\hline
\end{tabular}

Table 3. Optical properties of fluoropolymers

\section{Conclusion}

We prepared novel fluorinated amorphous polymers, trifluoroaryloxyether polymers, and investigated their chemical and optical properties. We demonstrated that these fluoropolymers could be used as TARC materials for 193-nm lithography due to their excellent dissolution behavior and optical properties. We showed that these fluoropolymers have the advantages of excellent optical transmittance, $\mathrm{k}$ value $<0.01$ at $193 \mathrm{~nm}$ (wavelength), and a water-soluble and low refractive index, i.e. less than $n=1.4$. They offer excellent potential as TARC materials.

\section{Acknowledments}

The authors would like to thank Kazuko Aoki, Yousuke Kishikawa and Yoshito Andou of the chemical division for synthesizing the polymers and monomers and polymer characterization.

\section{References}

1. R. R. Kunz, T. M. Bloomstein, D. E. Hardy, R. B. Goodman, D. K. Downs, and J. E. Curtin, "Outlook for 157-nm resist design," Proc. SPIE 3678, 13 (1999).; T. H. Fedynyshyn, R. R. Kunz, S. O. Doran, R. B. Goodman, M. L. Lind, and J. E. Curtin, "Prospects for Using Existing Resists for Evaluating 157-nm Imaging Systems," Proc. SPIE 3999, 335-346 (2000); T. H. Fedynyshyn, R. R. Kunz, R. F. Sinta, M. Sworin, W. A. Mowers, R. B. Goodman, and S. P. Doran, "High Resolution Fluorocarbon Based Resist for 157-nm Lithography," Proc. SPIE 4345, 296 (2001).; T. H. Fedynyshyn, W. A. Mowers, R. R. Kunz, R. F. Sinta, M. Sworin and R. B. Goodman, "High
Resolution Fluorocarbon Based Resist for 157-nm Lithography," Proc. SPIE 4690, 29 (2002).

2. H. Ito, Hoa D. Truong, M. Okazaki, D. C. Miller, N. Fender, G. Breyta, P. J. Brock, G. M. Wallraff, C. E. Larson, R. D. Allen, "Aliphatic platforms for the design of $157 \mathrm{~nm}$ chemically amplified resists," Proc. SPIE 4690, 18 (2002).; H. Ito, G. M. Wallraff, P. Brock, N. Fender, W. D. Hinsberg, H. Truong, G. Breyta, D. C. Miller, M. H. Sherwood, and R. D. Allen, "Polymer design for $157 \mathrm{~nm}$ chemically amplified resists," Proc. SPIE 4345, 273 (2001).

3. V. R. Vohra, K. Douki, Y. Kwark, X. Liu, C. K. Ober, and Y. C. Bae, "Highly Transparent Resist Platforms for $157 \mathrm{~nm}$ Microlithography: An Update," Proc. SPIE 4690, 84 (2002) ; Y. C. Bae, K. Douki, T. Yu, J. Dai, D. Schmaljohann, S. H. Kang, K. H. Kim, H. Koerner, W. Conley, D. Miller, R. Balasubramania, S. Holl, and C. K. Ober, "Rejuvenation of $248 \mathrm{~nm}$ Resist Backbones for $157 \mathrm{~nm}$ Lithography," J. Photopolymer Sci. Technol. 14, 613 (2001).; D. Schmaljohann, Y. C. Bae, G. L. Weibel, A. H. Hamad, and C. K. Ober, "Design Strategies for $157 \mathrm{~nm}$ Single-Layer Photoresists: Lithographic Evaluation of a Poly( $\alpha$-trifluoromethyl vinyl alcohol) Copolymer," Proc. SPIE 3999, 330 (2000)

4. Y. Uetani, K. Hashimoto, Y. Miya, I. Yoshida, M. Takigawa, and R. Hanawa, "Effect of fluorinated monomer unit introduction to $\mathrm{KrF}$ resin system in F2lithography," Proc. SPIE 4345, 379 (2001).

5. K. Patterson, M. Yamachika, R. Hung, C. Brodsky, S. Yamada, M. Somervell, B. Osborn, D. Hall, G. Dukovic, J. Byers, W. Conley, and C. G. Willson, "Polymers for $157 \mathrm{~nm}$ Photoresist Applications: A Progress Report," Proc. SPIE 3999, 365 (2000); R. J. Hung, H. V. Tran, B. C. Trinque, T. Chiba, S. Yamada, D. P. Sanders, E. F. Connor, R. H. Grubbs, J. Klopp, J. M. J. Frechet, B. H. Thomas, G. J. Shafer, D. D. DesMarteau, W. Conley, and C. G. Willson, "Resist Materials for 157 nm Microlithography: An Update," Proc. SPIE 4345, 385 (2001); B. C. Trinque, B. P. Osborn, C. R. Chambers, Y. Hsieh, S. Corry, T. Chiba, R. J. Hung, H. V. Tran, P. Zimmerman, D. Miller, W. Conley, and C. G. Willson, "Advances in Resists for $157 \mathrm{~nm}$ Microlithography," Proc. SPIE 4690, 58 (2002).; R. R. Dammel, R. 
Sakamuri, S. Lee, M. D. Rahman, T. Kudo, A. Romano, L. Rhodes, J. Lipian, C. Hacker and D. A. Barnes, "Cycloolefin/Cyanoacrylate (COCA) Copolymers For $193 \mathrm{~nm}$ And $157 \mathrm{~nm}$ Lithography," Proc. SPIE 4690, 101 (2002);

6. M. K. Crawford, A. E. Feiring, J. Feldman, R. H. French, M. Periyasamy, F. L. Schadt III, R. J. Smalley, F. C. Zumsteg, R. R. Kunz, V. Rao, L. Liao, and S. M. Holl, "New Materials for $157 \mathrm{~nm}$ Photoresists: Characterization and Properties," Proc. SPIE 3999, 357 (2000); M. K. Crawford, A. E. Feiring, J. Feldman, R. H. French, V. A. Petrov, F. L. Schadt III, R. J. Smalley, and F. C. Zumsteg, "157 nm Imaging Using Thick Single Layer Resists," Proc. SPIE 4345, 428 (2001).

7. T. Hagiwara, T. Furukawa, T. Itani, K. Fujii, T. Ishikawa, M. Koh, T. Kodani, T. Moriya, T. Yamashita, T. Araki, M. Toriumi, H. Aoyama, "Characterization of TFE/norbornene-based fluoropolymer resist for 157-nm lithography," Proc. SPIE 5376, 159 (2004)

M. Toriumi, N. Shida, H. Watanabe, T. Yamazaki, S. Ishikawa, and T. Itani, "Fluoropolymer Resists for 157-nm Lithography," Proc. SPIE 4690, 191 (2002); N. Shida, H. Watanabe, T. Yamazaki, S. Ishikawa, M. Toriumi, and T. Itani, "157-nm Single Layer Resists Based on Advanced Fluorinated Polymers," Proc. SPIE 4690, 497 (2002); M. Toriumi, S. Ishikawa, S. Miyoshi, T. Naito, T. Yamazaki, M. Watanabe, and T. Itani, "Resist materials for $157-\mathrm{nm}$ lithography," Proc. SPIE 4345, 371 (2001).

8. S. Kodama, I. Kaneko, Y. Takebe, S. Okada, Y. Kawaguchi, N. Shida, S. Ishikawa, M. Toriumi, and T. Itani, "Synthesis of Novel Fluoropolymer for $157 \mathrm{~nm}$ Photoresists," Prd6. SPIE 4690, 76 (2002); Y. Takebe, M. Eda, S. Okada, O. Yokokoji, S. Irie, A. Otoguro, K. Fujii, T. Itani" A new monocyclic fluropolymer structure for 157-nm photoresists," Proc. SPIE 5376, 151(2004)

9. M. Koh, T. Ishikawa, T. Araki, H. Aoyama, T. Yamashita, T. Yamazaki, H. Watanabe, M. Toriumi, and T. Itani, "The study on dry etch resistance of Fluorine functionalized polymers," Proc. SPIE 4690, 486 (2002).

10. M. Koh, T. Ishikawa, T. Araki, H. Aoyama, T. Yamashita, T. Yamazaki, H. Watanabe, M. Toriumi, and T. Itani, "Synthesis of novel fluorinated norbornene derivatives for $157 \mathrm{~nm}$ application," Proc. SPIE 5039, 589 (2003).

11. M. Toriumi, M. Koh, T. Ishikawa, T. Araki, H. Aoyama, T. Yamashita, T. Yamazaki, H. Watanabe, and T. Itani, "Novel Main-chain-Fluorinated Polymers for 157-nm Photoresists," Proc. SPIE 5039, 53 (2003).

12. T. Ishikawa, T. Kodani, M. Koh, T. Moriya, T. Araki, H. Aoyama, T. Yamashita, M. Toriumi, T. Hagiwara, T. Furukawa, T. Itani, K. Fujii, " The dissolution behavior of tetrafluoroethylene-based fluoropolymers for 157-nm resist materials," Proc. SPIE 5376, 169 (2004).

13. T. Shimizu, S.Morita, T.Araki, Y.Tanaka, International Conference on Fluorine Chemistry '99, Tokyo, May 10 (1999). T.Shimizu, S.Morita, T.Araki, Y.Tanaka, presented at the 16thInternational Symposium on Fluorine Chemistry, Durham, England, July 2000; 2P-89.

14. M. Toriumi, T. Ohfuji, M. Endo, and H. Morimoto, "Swelling Analysis of Methacrylate Polymers in Aqueous Alkaline Developer," $J$. Photopolymer Sci. Technol. 12, 545 (1999); M. Toriumi, S. Ishikawa, and T. Itani, "Resist polymer dissolution study using Quartz Crystal Microbalance method," in Forefront of Lithographic Materials Research, Society of Plastic Engineers, Mid Hudson Section, pp. 271-281 (2001); M. Toriumi, T. Itani, J. Yamashita, T. Sekine, and K. Nakatani, "Dissolution characteristics of resist polymers studied by the Quartz Crystal Microbalance transmission-line analysis and the pKa acidity analysis", Proc. SPIE 4690904 (2002) and references therein.

15. T. Yamashita, T. Ishikawa, T. Yoshida, T. Hayami, T. Araki, H. Aoyama, T. Hagiwara, T. Itani, K. Fujii, " Synthesis of fluorinated materials for 193-nm immersion lithography and 157-nm lithography," Proc. SPIE 5753, 564 (2005). T. Yamashita, T. Ishikawa, T. Yoshida, T. Hayami, T. Araki, H. Aoyama, T. Hagiwara, T. Itani, K. Fujii," Synthesis of fluorinated materials for 193-nm immersion lithography and 157-nm lithography," J. Photopolymer Sci. Technol. 18, 631 (2005).

16. T. Yamashita, T. Ishikawa, T. Yoshida, T. Hayami, H. Aoyama, "Novel fluorinated polymers for application in 193-nm lithography and 193-nm immersion lithography "Proc. SPIE 6153-81 (2006). 